\title{
Flat Affect
}

National Cancer Institute

\section{Source}

National Cancer Institute. Flat Affect. NCI Thesaurus. Code C117196.

The absence of emotional expressiveness. 